\title{
LINEAR FACTORIZATION OF CONICAL POLYNOMIALS OVER CERTAIN NONASSOCIATIVE ALGEBRAS
}

BY

\section{J. LEPOWSKY (1)}

\begin{abstract}
Conical polynomials are defined as certain polynomials in quadratic elements of the universal enveloping algebra of a semisimple symmetric Lie algebra over a field of characteristic zero. These polynomials were used in an earlier paper to describe the conical vectors in certain induced modules. Here it is shown that when the base field is extended to a certain type of nonassociative algebra, the conical polynomials can be factored "linearly". One such nonassociative algebra is discussed in detail-an (alternative) composition algebra intimately related to the structure of the Lie algebra and studied earlier by B. Kostant in the context of real semisimple Lie algebras. The linear factorization leads in a later paper to an extension of the earlier work on conical vectors in induced modules.
\end{abstract}

1. Introduction. In [3(b)], certain conical vectors in induced modules for a semisimple symmetric Lie algebra $g$ were found. These vectors were expressed by means of polynomials $\zeta_{l}$ in certain quadratic elements $q_{-\alpha}$ and $q_{-2 \alpha}$ of the universal enveloping algebra of $g$ (see the Introduction and Theorem 10.1 of [3(b)] ). We call the $\zeta_{l}$ "conical polynomials" (cf. §4). They are products of factors of the form $q_{-\alpha}$ and $q_{-\alpha}^{2}+j^{2} q_{-2 \alpha}$ ( $j$ an integer). In order to prove the main result (Theorem 10.1) of [3(b)], we had to use "transfer principles" to reduce to a special case in which the double root space $g^{2 \alpha}$ is one-dimensional. The base field could also be assumed algebraically closed. The necessary computations could be performed in this case because $q_{-2 \alpha}$ has a natural square root $r_{-2 \alpha} \in$ $g^{-2 \alpha}$, permitting the quadratic expressions $q_{-\alpha}^{2}+j^{2} q_{-2 \alpha}$ to factor "linearly" as $\left(q_{-\alpha}+(-1)^{1 / 2} j r_{-2 \alpha}\right)\left(q_{-\alpha}-(-1)^{1 / 2} j r_{-2 \alpha}\right)$. (See [3(b)].) It is natural to ask for a direct proof (not requiring the transfer principles) of the main result of [3(b)], and we are led to try to adjoin a square root of $-q_{-2 \alpha}$ to the polynomial algebra $k\left[q_{-\alpha}, q_{-2 \alpha}\right]$, to be used the way $(-1)^{1 / 2} r_{-2 \alpha}$ was used when $\operatorname{dim} g^{2 \alpha}=1$. In this paper, we describe a general method for extending the base field, not

Received by the editors January 14, 1975.

AMS (MOS) subject classifications (1970). Primary 17B05, 17B20, 17D05, 17B10; Secondary 22E45, $17 \mathrm{~B} 35$.

Key words and phrases. Conical polynomials, conical vectors, semisimple symmetric Lie algebras, alternative algebras, composition algebras, Cayley-Dickson algebras.

(1) Partially supported by NSF GP 33893. 
to a larger field, but to a certain type of nonassociative algebra, so that a square root $k_{-2 \alpha}$ of $-q_{-2 \alpha}$ can be constructed over the extension (see Proposition 4.3). The subalgebra of the extended universal enveloping algebra generated by $q_{-\alpha}$ and $\kappa_{-2 \alpha}$ turns out to be the commutative, associative polynomial algebra generated by these elements (see Theorem 4.6), so that the conical polynomials can be factored linearly just as when $\operatorname{dim} g^{2 \alpha}=1$ (Theorem 4.7).

Two nonassociative algebras which can be used for this extension are a certain Jordan algebra and a certain Clifford algebra. The most interesting, though, is a composition algebra $c_{\varphi}$ (based on a given restricted root $\varphi$ ) which is the subject of $\S \S 2$ and 3. The algebra $c_{\varphi}$ is defined in $\S 2$ using a choice of $B_{\theta}$-nonisotropic vector $e_{0} \in g^{\varphi}$ (see $\S 2$ ), and its elementary properties are proved there. In $\S 3$, we show that $c_{\varphi}$ is an (alternative) composition algebra and hence a Cayley-Dickson algebra, that $c_{\varphi}$ is independent of $e_{0}$ up to isomorphism, and that $c_{\varphi}$ is isomorphic to $c_{-\varphi}$. For real semisimple Lie algebras with Cartan decomposition, the existence and properties of $c_{\varphi}$ are known, and $c_{\varphi}$ is a division algebra in this case (see [2(a), p. 635, Remark 4]). This is implicit in B. Kostant's proof of the Kostant-Mostow double transitivity theorem, and our treatment is based on his; cf. [2(b), §2.1] and [3(b), §4]. The alternativity of $c_{\varphi}$ (Theorem 3.1) is intimately related to Kostant's proof of the double transitivity result.

Unfortunately, we have been unable to use these ideas to carry out our original goal of giving a new direct proof of Theorem 10.1 of [3(b)] for the cases $\operatorname{dim} g^{2 \alpha}>1$. But the linear factorization of the conical polynomials has led us to a considerable extension of that theorem, to appear later [3(c)]. (Incidentally, using another approach, we have found a direct proof of Theorem 10.1 of $[3(b)]$, also to appear later [3(c)] .)

We refer the reader to [4] and [1] for information on alternative and composition algebras, and other nonassociative algebra concepts that we shall use.

2. Definition and elementary properties of $c_{\varphi}$. We shall work in the setting of $[3(b)]$. Let $(g, \theta)$ be a semisimple symmetric Lie algebra with symmetric decomposition $g=\notin \oplus p$ over a field $k$ of characteristic zero, and let $a$ be a splitting Cartan subspace of $k$. Let $m$ be the centralizer of $a$ in $E$ and $\Sigma \subset a^{*}$ ( ${ }^{*}$ denotes dual) the set of restricted roots of $g$ with respect to a. Also let $B$ be the Killing form of $g$ and $B_{\theta}$ the nonsingular symmetric bilinear form on $g$ defined by: $B_{\theta}(x, y)=-B(x, \theta y)$ for all $x, y \in g$. Then the root space decomposition $g=g^{0} \oplus \amalg_{\varphi \in \Sigma} g^{\varphi}$, with $g^{0}=m \oplus a$, is $B_{\theta}$-orthogonal, and $B_{\theta}$ is nonsingular on $m$, $a$ and each $g^{\varphi}$. Denote by $(\cdot, \cdot)$ the resulting nonsingular symmetric bilinear form on $a^{*}$. For each $\varphi \in a^{*}$, define $x_{\varphi} \in a$ to be the image of $\varphi$ under the canonical isometry from $a^{*}$ to $a$. If $(\varphi, \varphi) \neq 0$ (for example, if $\varphi \in$ $\Sigma)$, define $h_{\varphi}=2 x_{\varphi} /(\varphi, \varphi) \in a$, so that $\varphi\left(h_{\varphi}\right)=2$. Then $h_{2 \varphi}=1 / 2 h_{\varphi}$ for all $\varphi \in \Sigma$ (even if $2 \varphi \notin \Sigma$ ). 
Fix $\varphi \in \Sigma$. The space $g^{2 \varphi} \oplus g^{-2 \varphi}$ (which might be zero) is $\theta$-stable. Let $p_{\varphi}^{2}$ be its intersection with $p$, so that the map

$$
i_{\varphi}: g^{2 \varphi} \rightarrow p_{\varphi}^{2}, \quad x \mapsto x-\theta x
$$

is a linear isomorphism. Also, $i_{\varphi}$ is an isometry from $2 B_{\theta} \lg ^{2 \varphi} \times g^{2 \varphi}$ to $B_{\theta} \mid p_{\varphi}^{2} \times p_{\varphi}^{2}$.

Define $c_{\varphi}=k h_{2 \varphi} \oplus p_{\varphi}^{2}$. This is an orthogonal direct sum of nonsingular spaces for $B_{\theta}$ and $B$. These two bilinear forms are equal on $c_{\varphi}$ (and on all of $p$ ), and are nonsingular on $C_{\varphi}$.

Fix a $B_{\theta}$-nonisotropic vector $e_{0} \in g^{\varphi}$. Then by [3(a), Lemma 3.3],

$$
\left[e_{0},-\theta e_{0}\right]=B_{\theta}\left(e_{0}, e_{0}\right) x_{\varphi}=1 / 2(\varphi, \varphi) B_{\theta}\left(e_{0}, e_{0}\right) h_{\varphi} .
$$

Let $c_{0}=1 / 2(\varphi, \varphi) B_{\theta}\left(e_{0}, e_{0}\right)$ and $f_{0}=-\theta e_{0}$, so that $\left[e_{0}, f_{0}\right]=c_{0} h_{\varphi}$, and $c_{0} \neq 0$.

Let $K$ be an extension field of $k$ such that $c_{0}$ has a square root in $K$. (For example, $K$ may be an algebraic closure of $k$.) Define $g_{K}=g \otimes_{k} K, \mathfrak{E}_{K}=\mathfrak{E}$ $\otimes_{k} K$, etc., and denote again by $\theta$ the $K$-linear extension of $\theta$ to $g_{K}$. Then $\left(g_{K}, \theta\right)$ is a semisimple symmetric Lie algebra over $K, a_{K}$ is a splitting Cartan subspace of $p_{K}$, etc.

Regard $e_{0}$ and $f_{0}$ as elements of $g_{K}$, and define $e_{\varphi} \in\left(g^{\varphi}\right)_{K}$ and $f_{\varphi} \in$ $\left(g^{-\varphi}\right)_{K}$ by the formulas $e_{\varphi}=c_{0}^{-1 / 2} e_{0}$ and $f_{\varphi}=c_{0}^{-1 / 2} f_{0}$. Then $f_{\varphi}=-\theta e_{\varphi}$, and $\left[e_{\varphi}, f_{\varphi}\right]=h_{\varphi}$. Inside the three-dimensional simple $K$-subalgebra of $g_{K}$ spanned by $h_{\varphi}, e_{\varphi}$ and $f_{\varphi}$, let

$$
y_{\varphi}=1 / 2\left(e_{\varphi}+f_{\varphi}\right) \in p_{K} \quad \text { and } \quad z_{\varphi}=1 / 2\left(e_{\varphi}-f_{\varphi}\right) \in \mathbb{E}_{\cdot K} .
$$

We are now ready to define our nonassociative algebra structure on $c_{\varphi}$. First define such a structure on $\left(c_{\varphi}\right)_{K}$ as follows: For all $a, b \in\left(c_{\varphi}\right)_{K}$, let

$$
a \circ b=\left[\left[y_{\varphi}, a\right],\left[z_{\varphi}, b\right]\right] \text {. }
$$

We shall see below that $a \circ b \in\left(c_{\varphi}\right)_{K}$. Assuming this, it is clear that if $a, b \in$ $c_{\varphi}$, then $a \circ b \in c_{\varphi}$. In fact, $a \circ b$ is given by the following expression over $k$ :

$$
\begin{aligned}
a \circ b=\left(4 c_{0}\right)^{-1}\left(\left[\left[e_{0}, a\right],\left[e_{0}, b\right]\right]-\left[\left[f_{0}, a\right],\left[f_{0}, b\right]\right]\right. \\
\left.-\left[\left[e_{0}, a\right],\left[f_{0}, b\right]\right]+\left[\left[f_{0}, a\right],\left[e_{0}, b\right]\right]\right) .
\end{aligned}
$$

The following two propositions will be proved together:

Proposition 2.1. The space $c_{\varphi}$ is closed under the product $a \circ b$, and $h_{2 \varphi}$ is the identity element of the resulting nonassociative algebra. The conjuga- 
tion $a \mapsto \bar{a}$ of $c_{\varphi}$ with respect to the decomposition $c_{\varphi}=k h_{2 \varphi} \oplus p_{\varphi}^{2}$ is an antiautomorphism of $c_{\varphi}$. Let $N$ and $T$ be the corresponding norm and trace functions on $\mathrm{c}_{\varphi}$, i.e.,

$$
a \circ \bar{a}=\bar{a} \circ a=N(a) h_{2 \varphi} \quad(N(a) \in k)
$$

and

$$
a+\bar{a}=T(a) h_{2 \varphi} \quad(T(a) \in k)
$$

for all $a \in c_{\varphi}$. Then

$$
N(a)=(\varphi, \varphi) B_{\theta}(a, a)=(\varphi, \varphi) B(a, a)
$$

and

$$
T(a)=2(\varphi, \varphi) B_{\theta}\left(h_{2 \varphi}, a\right)=2(\varphi, \varphi) B\left(h_{2 \varphi}, a\right) .
$$

In particular, $N$ is a nondegenerate quadratic form on $\mathrm{c}_{\varphi}$ with associated bilinear form $(\varphi, \varphi) B_{\theta}(\cdot, \cdot)$, and $T$ is a linear form on $c_{\varphi}$ with kernel $\hat{\beta}_{\varphi \cdot}^{2}$ Also, if $x \in$ $g^{2 \varphi}$, then

$$
N\left(i_{\varphi} x\right)=2(\varphi, \varphi) B_{\theta}(x, x)
$$

Proposition 2.2. Suppose $k=K$, and let $g_{*}=\left[f_{\varphi}, g\right]$ for all $g \in g$. For all $x, y \in g^{2 \varphi}$,

$$
\left(i_{\varphi} x\right) \circ\left(i_{\varphi} y\right)=-2(\varphi, \varphi) B_{\theta}(x, y) h_{2 \varphi}-1 / 4 i_{\varphi}\left[x_{*}, y_{*}\right]
$$

Proof. In proving these two propositions, it is clearly sufficient to assume that $k=K$, and that this field is algebraically closed. First note that for all $a$, $b \in c_{\varphi}$,

$$
a \circ b=1 / 4\left(\left[a^{*}, b^{*}\right]-\left[a_{*}, b_{*}\right]-\left[a^{*}, b_{*}\right]+\left[a_{*}, b^{*}\right]\right),
$$

where $g^{*}$ denotes $\left[e_{\varphi}, g\right]$, for all $g \in g$. Since $\left(h_{2 \varphi}\right)^{*}=-e_{\varphi}$ and $\left(h_{2 \varphi}\right)_{*}=f_{\varphi}$, it is clear that $h_{2 \varphi} \circ h_{2 \varphi}=h_{2 \varphi}$. For all $a \in \mathfrak{p}^{2 \varphi}$,

$$
h_{2 \varphi} \circ a=1 / 4\left(-a^{* *}-a_{* *}+\left(a_{*}\right)^{*}+\left(a^{*}\right)_{*}\right) \text {. }
$$

Writing $a=i_{\varphi} x=x-\theta x$ for $x \in g^{2 \varphi}$, we have

$$
h_{2 \varphi} \circ a=1 / 4\left((\theta x)^{* *}-x_{* *}+\left(x_{*}\right)^{*}-\left((\theta x)^{*}\right)_{*}\right) .
$$

By the representation theory of the three-dimensional simple Lie algebra spanned by $h_{\varphi}, e_{\varphi}$ and $f_{\varphi}$, we have $\left((\theta x)^{*}\right)_{*}=4 \theta x$ and $\left(x_{*}\right)^{*}=4 x$ (cf. [3(b), Lemma 4.15]). Also, $x_{* *} \in \mathrm{m}$ by [3(b), Lemma 4.11], so that $x_{* *}=\theta x_{* *}=(\theta x)^{* *}$. 
Hence $h_{2 \varphi} \circ a=x-\theta x=a$. Similarly, $a \circ h_{2 \varphi}=a$ for all $a \in \mathcal{p}_{\varphi}^{2}$, and so $h_{2 \varphi} \circ a=a \circ h_{2 \varphi}=a$ for all $a \in c_{\varphi}$.

Now let $x, y \in g^{2 \varphi}$. Then

$$
\begin{aligned}
\left(i_{\varphi} x\right) & \circ\left(i_{\varphi} y\right)=(x-\theta x) \circ(y-\theta y) \\
& =1 / 4\left(\left[(\theta x)^{*},(\theta y)^{*}\right]-\left[x_{*}, y_{*}\right]+\left[(\theta x)^{*}, y_{*}\right]-\left[x_{*},(\theta y)^{*}\right]\right) \\
& =1 / 4\left(-\left[x_{*}, y_{*}\right]+\theta\left[x_{*}, y_{*}\right]+\left[(\theta x)^{*}, y_{*}\right]-\left[x_{*},(\theta y)^{*}\right]\right) \\
& =-1 / 4 i_{\varphi}\left[x_{*}, y_{*}\right]+1 / 4\left(\left[(\theta x)^{*}, y_{*}\right]-\left[x_{*},(\theta y)^{*}\right]\right)
\end{aligned}
$$

(note that $\left.\left[x_{*}, y_{*}\right] \in g^{2 \varphi}\right)$. Let $C(x, y)$ be the second term of this last expression. Then $C$ is bilinear in $x$ and $y$, and $C(x, y)=C(y, x)$, i.e., $C$ is symmetric. But

$$
\begin{aligned}
C(x, x) & =1 / 4\left(\left[(\theta x)^{*}, x_{*}\right]-\left[x_{*},(\theta x)^{*}\right]\right)=-1 / 2\left[x_{*},(\theta x)^{*}\right] \\
& =1 / 2\left[x_{*}, \theta x_{*}\right]=-1 / 2 B_{\theta}\left(x_{*}, x_{*}\right) x_{\varphi} \quad \text { (by [3(a), Lemma 3.3]) } \\
& =1 / 2 B\left(x_{*},-(\theta x)^{*}\right) x_{\varphi}=1 / 2 B\left(\left(x_{*}\right)^{*}, \theta x\right) x_{\varphi} \quad \text { (since } B \text { is invariant) } \\
& =2 B(x, \theta x) x_{\varphi}=-(\varphi, \varphi) B_{\theta}(x, x) h_{\varphi}=-2(\varphi, \varphi) B_{\theta}(x, x) h_{2 \varphi} .
\end{aligned}
$$

Since $-2(\varphi, \varphi) B_{\theta}(x, y) h_{2 \varphi}$ is a symmetric bilinear expression in $x$ and $y$ which agrees with $C(x, y)$ when $x=y$, it must also agree for arbitrary $x, y \in g^{2 \varphi}$, by polarization. Thus

$$
\left(i_{\varphi} x\right) \circ\left(i_{\varphi} y\right)=-1 / 4 i_{\varphi}\left[x_{*}, y_{*}\right]-2(\varphi, \varphi) B_{\theta}(x, y) h_{2 \varphi}
$$

for all $x, y \in g^{2 \varphi}$, proving Proposition 2.2, as well as the fact that $\mathrm{c}_{\varphi}$ is closed under the nonassociative multiplication.

To show that the conjugation $a \mapsto \bar{a}$ of $c_{\varphi}$ is an antiautomorphism, it is sufficient to check that $\left(\left(i_{\varphi} x\right) \circ\left(i_{\varphi} y\right)\right)^{-}=\left(i_{\varphi} y\right) \circ\left(i_{\varphi} x\right)$ for all $x, y \in g^{2 \varphi}$. But this is immediate from Proposition 2.2.

Let $a=c h_{2 \varphi}+i_{\varphi} x \in \mathrm{c}_{\varphi}$, where $c \in k$ and $x \in g^{2 \varphi}$. Then

$$
a \circ \bar{a}=c^{2} h_{2 \varphi}-\left(i_{\varphi} x\right) \circ\left(i_{\varphi} x\right)=\left(c^{2}+2(\varphi, \varphi) B_{\theta}(x, x)\right) h_{2 \varphi} \text {. }
$$

But $B_{\theta}(x, x)=1 / 2 B_{\theta}\left(i_{\varphi} x, i_{\varphi} x\right)$, and $B_{\theta}\left(h_{2 \varphi}, h_{2 \varphi}\right)=1 /(\varphi, \varphi)$. Hence

$$
a \circ \bar{a}=(\varphi, \varphi)\left(B_{\theta}\left(c h_{2 \varphi}, c h_{2 \varphi}\right)+B_{\theta}\left(i_{\varphi} x, i_{\varphi} x\right)\right) h_{2 \varphi}=(\varphi, \varphi) B_{\theta}(a, a) h_{2 \varphi} \text {, }
$$

and so $N(a)=(\varphi, \varphi) B_{\theta}(a, a)$. The rest of Proposition 2.1 is clear. Q.E.D. 
3. The alternativity of $c_{\varphi}$.

THEOREM 3.1. The nonassociative algebra $\mathrm{c}_{\varphi}$ is alternative.

Proof. We may, and do, assume that $k$ is algebraically closed. Since $c_{\varphi}$ has an antiautomorphism (see Proposition 2.1), it is sufficient to show that $a$ 。 $(a \circ b)=(a \circ a) \circ b$ for all $a, b \in c_{\varphi}$. We may clearly assume that $a, b \in p_{\varphi}^{2}$. By Proposition 2.2, what we must show is that for all $x, y \in g^{2 \varphi}$,

$$
\left(i_{\varphi} x\right) \circ\left(\left(i_{\varphi} x\right) \circ\left(i_{\varphi} y\right)\right)=-2(\varphi, \varphi) B_{\theta}(x, x)\left(i_{\varphi} y\right) .
$$

As in $\S 2$, write $g_{*}=\left[f_{\varphi}, g\right]$ for $g \in g$. Let $x, y \in g^{2 \varphi}$. Since $\left[x_{*}, y\right]=$ $\left[x, y_{*}\right]=0$, we have $\left[x_{*}, y\right]_{*}=\left[x, y_{*}\right]_{*}=0$, i.e., $\left[x_{*}, y_{*}\right]=-\left[x_{* *}, y\right]=$ $-\left[x, y_{* *}\right]$. Thus by Proposition 2.2,

$$
\left(i_{\varphi} x\right) \circ\left(i_{\varphi} y\right)=-2(\varphi, \varphi) B_{\theta}(x, y) h_{2 \varphi}+1 / 4 i_{\varphi}\left[x_{* *}, y\right],
$$

and

$$
\begin{aligned}
\left(i_{\varphi} x\right) \circ\left(\left(i_{\varphi} x\right) \circ\left(i_{\varphi} y\right)\right)= & -2(\varphi, \varphi) B_{\theta}(x, y)\left(i_{\varphi} x\right) \\
& -1 / 2(\varphi, \varphi) B_{\theta}\left(x,\left[x, y_{* *}\right]\right) h_{2 \varphi}+(1 / 16) i_{\varphi}\left[x_{* *},\left[x_{* *}, y\right]\right] .
\end{aligned}
$$

But the middle term on the right is zero. Indeed,

$$
B_{\theta}\left(x,\left[x, y_{* *}\right]\right)=-B\left(\theta x,\left[x, y_{* *}\right]\right)=B\left([x, \theta x], y_{* *}\right),
$$

and this is zero because $[x, \theta x] \in F($ since $\theta[x, \theta x]=-[x, \theta x])$, while $y_{* *} \in$ $\mathfrak{m}$, by [3(b), Lemma 4.11]. Hence it is sufficient to prove:

LEMMA 3.2. For all $x, y \in \varphi^{2 \varphi}$,

$$
\left[x_{* *},\left[x_{* *}, y\right]\right]=32(\varphi, \varphi)\left(B_{\theta}(x, y) x-B_{\theta}(x, x) y\right) .
$$

Proof. Write $g^{*}=\left[e_{\varphi}, g\right]$ for $g \in g$. Let $u=y_{* *}$ and $v=x_{* *}$, so that in the notation of [3(b), Lemma 4.14], $u, v \in \mathrm{m}_{2}$. We also have $u^{*}=6 y_{*}$ and $u^{* *}=24 y$ (cf. [3(b), Lemma 4.15]), and similarly, $v^{* *}=24 x$.

By [3(b), Lemmas 4.18 and 4.19], applied to $u$ and $v$ in place of $x$ and $y$, respectively, we have

$$
\left[[u, v], v^{* *}\right]=\left[[u, v]_{0}, v^{* *}\right]+\left[[u, v]_{2}, v^{* *}\right]=1 / 2\left[[u, v]_{0}, v^{* *}\right],
$$

where the subscript notation is defined in [3(b)] before Lemma 4.18. Suppose that $B_{\theta}\left(u^{* *}, v^{* *}\right)=0$ and that $B_{\theta}\left(v^{* *}, v^{* *}\right)=1 / 2(\varphi, \varphi)$. Then by [3(b), Lem$\operatorname{ma} 4.22],\left[[u, v], v^{* *}\right]=-(1 / 36) u^{* *}$.

Reformulating in terms of $x$ and $y$, we have that if $B_{\theta}(x, y)=0$ and $(24)^{2} B_{\theta}(x, x)=1 / 2(\varphi, \varphi)$, then $\left[\left[y_{* *}, x_{* *}\right], x\right]=-(1 / 36) y$, i.e., 


$$
\left[\left[y_{* *}, x_{* *}\right], x\right]=-32(\varphi, \varphi) B_{\theta}(x, x) y .
$$

This last equation is clearly true whenever $B_{\theta}(x, y)=0$ and $B_{\theta}(x, x) \neq 0$. The lefthand side equals

$$
\left[\left[y_{* *}, x\right], x_{* *}\right]+\left[y_{* *},\left[x_{* *}, x\right]\right]=\left[\left[y, x_{* *}\right], x_{* *}\right]-\left[y_{* *},\left[x_{*}, x_{*}\right]\right]
$$

(see the beginning of the proof of Theorem 3.1)

$$
=\left[x_{* *},\left[x_{* *}, y\right]\right] \text {. }
$$

Thus under our assumptions,

$$
\left[x_{* *},\left[x_{* *}, y\right]\right]=-32(\varphi, \varphi) B_{\theta}(x, x) y .
$$

Suppose now that $B_{\theta}(x, x) \neq 0$ but that $y$ is arbitrary. Applying the above to $B_{\theta}(x, x) y-B_{\theta}(x, y) x$ (which is $B_{\theta}$-orthogonal to $x$ ) in place of $y$, we get

$$
\left[x_{* *},\left[x_{* *}, y\right]\right]=32(\varphi, \varphi)\left(B_{\theta}(x, y) x-B_{\theta}(x, x) y\right) .
$$

But for each fixed $y$, the two sides of this equation are polynomial maps (in $x$ ) from $g^{2 \varphi}$ into itself which agree on the Zariski dense subset on which $B_{\theta}(x, x) \neq$ 0 . Hence the equation holds for all $x, y \in g^{2 \varphi}$, and so Lemma 3.2 and Theorem 3.1 are proved. Q.E.D.

Using E. Artin's theorem that the subalgebra generated by any two elements of an alternative algebra is associative (see [4, p. 29, Theorem 3.1]), it is now straightforward to show that the norm function $N$ of $c_{\varphi}$ permits composition, i.e., is multiplicative (see $[4$, p. 58]). By Proposition $2.1, N$ is a nondegenerate quadratic form on $c_{\varphi}$. Recalling that a composition algebra is a nonassociative algebra with a nondegenerate quadratic form permitting composition (cf. [4, p. 73] or [1, pp. 162-163]), we now have:

THEOREM 3.3. The nonassociative algebra $\mathrm{c}_{\varphi}$ is a composition algebra.

By the standard structure theorem for composition algebras (see either [4, p. 73, Theorem 3.25] or [1, p. 164, Theorem 5]), $c_{\varphi}$ is built up by the CayleyDickson construction from $k$. That is:

COROLlary 3.4. The dimension of $c_{\varphi}$ is either $1,2,4$ or 8 , and one of the following holds:

(i) $c_{\varphi}=k$;

(ii) $\operatorname{dim} c_{\varphi}=2$, and $\hat{p}_{\varphi}^{2}$ is spanned by an element whose square is in $k$, so that $c_{\varphi}$ is associative and commutative;

(iii) $c_{\varphi}$ is a 4-dimensional quaternion algebra with standard involution over $k$, so that $\mathrm{c}_{\varphi}$ is associative but not commutative; 
(iv) $c_{\varphi}$ is an 8-dimensional octonion algebra with standard involution over $k$, so that $\mathrm{c}_{\varphi}$ is neither associative nor commutative.

Since a nonassociative algebra obtained by the Cayley-Dickson construction from $k$ is determined up to isomorphism by its norm (see $[4, \mathrm{p} .70$, Theorem 3.23] and its proof), and since the norm on $\mathrm{C}_{\varphi}$ is as described in Proposition 2.1, we have:

THEOREM 3.5. The isomorphism class of $\mathrm{c}_{\varphi}$ is independent of the $B_{\theta}$-nonisotropic vector $e_{0} \in g^{\varphi}$ used to define it. Moreover, $c_{-\varphi}$ is isomorphic to $c_{\varphi}$.

4. Linear factorization of conical polynomials. For a nonassociative algebra $A$ (with 1 , over $k$ ), define the commutative nonassociative algebra $A^{+}$by means of the product $a \cdot b$ given by $a \cdot b=1 / 2(a b+b a)$, where $a, b \in A$ and $a b$ denotes multiplication in $A$. If $A$ is associative or even alternative, then $A^{+}$is a special Jordan algebra (see [1, p. 15, Theorem 3]).

Let $\omega$ be a symmetric bilinear form on a finite-dimensional vector space $V$. Then $k \oplus V$ becomes a Jordan algebra when we let $1 \in k$ be the identity and $v w$ $=\omega(v, w) \in k$ for all $v, w \in V$ (see [1, pp. 13-14]). It is called the Jordan algebra of $\omega$. Let $C(V, \omega)$ be the Clifford algebra of $\omega$. Then the Jordan algebra of $\omega$ naturally injects into the Jordan algebra $C(V, \omega)^{+}$(see $[1$, p. 261, Theorem 1]).

Call a nonassociative algebra $A$ together with a linear injection $j: k \oplus V \rightarrow$ $A \omega$-admissible if $j$ is an injection of the Jordan algebra of $\omega$ into $A^{+}$. This is equivalent to saying that $j(1)=1$ and for all $v \in V, j(v)^{2}=\omega(v, v) 1$ in $A$. Intuitively, this means that we can take the square root of $\omega$ in $A$. We shall say this in another way in Proposition 4.1 below. Note that the Jordan algebra of $\omega$ (with the identity map $j$ ) is $\omega$-admissible, as is $C(V, \omega)$ (with the natural map $j$ ).

Denote by $S(W)$ the symmetric algebra of a vector space $W$, and by $S^{r}(W)$ $(r=0,1,2, \ldots)$ the $r$ th symmetric power of $W$, so that $S(W)=\amalg S^{r}(W)$. The homogeneous quadratic polynomial function $v \mapsto \omega(v, v)$ on $V$ defines a canonical element $t_{\omega} \in S^{2}\left(V^{*}\right)$.

Suppose that $A$ is $\omega$-admissible, and identify $V$ with its image in $A$. Form the tensor product $S\left(V^{*}\right)_{A}=A \otimes_{k} S\left(V^{*}\right)$, and make $S\left(V^{*}\right)_{A}$ a nonassociative algebra by the natural definition: $(a \otimes s)\left(a^{\prime} \otimes s^{\prime}\right)=a a^{\prime} \otimes s s^{\prime}$, where $a, a^{\prime} \in A$ and $s, s^{\prime} \in S\left(V^{*}\right)$. (We are "extending scalars" from $k$ to the nonassociative algebra $A$.) Then $V \otimes V^{*} \simeq$ End $V$ is naturally embedded in $S\left(V^{*}\right)_{A}$. Let $\iota_{\omega}$ be the canonical element of $V \otimes V^{*}$ corresponding to the identity map in End $V$. The next result says that $t_{\omega} \in S^{2}\left(V^{*}\right)$ (which is esentially $\omega$ ) has a canonical square root, not in $S^{1}\left(V^{*}\right)=V^{*}$, but in $V \otimes V^{*} \subset A \otimes S^{1}\left(V^{*}\right)$ :

Proposition 4.1. In $S\left(V^{*}\right)_{A}, \iota_{\omega}^{2}=t_{\omega}$. 
Proof. Let $v_{1}, \ldots, v_{n}$ be an $\omega$-orthogonal basis of $V$, and $v_{1}^{*}, \ldots, v_{n}^{*}$ $\in V^{*}$ its dual basis. Then $\iota_{\omega}=\sum_{i=1}^{n} v_{i} \otimes v_{i}^{*}$. But since $A$ is $\omega$-admissible, $v_{i}^{2}=\omega\left(v_{i}, v_{i}\right) 1$ for all $i=1, \ldots, n$, and $v_{i} v_{j}+v_{j} v_{i}=2 \omega\left(v_{i}, v_{j}\right)=0$ for all $i \neq j$. Thus $i_{\omega}^{2}=\sum_{i=1}^{n} \omega\left(v_{i}, v_{i}\right)\left(v_{i}^{*}\right)^{2} \in S^{2}\left(V^{*}\right)$, since $S\left(V^{*}\right)$ is commutative. It is clear that this element is just $t_{\omega}$, since the two act as the same polynomial function on $V$. Q.E.D.

Now suppose that $\omega$ is nonsingular. Then $\omega$ induces a natural linear isomorphism $\xi_{\omega}: V^{*} \rightarrow V$, which extends to an algebra isomorphism $\xi_{\omega}: S\left(V^{*}\right) \rightarrow$ $S(V)$. Let $p_{\omega}=\xi_{\omega}\left(t_{\omega}\right)$, so that $p_{\omega} \in S^{2}(V)$. Since $1 \otimes \xi_{\omega}: A \otimes S\left(V^{*}\right) \rightarrow$ $A \otimes S(V)$ is an algebra isomorphism from $S\left(V^{*}\right)_{A}$ to $S(V)_{A}(=A \otimes S(V))$, we have, letting $\kappa_{\omega}=\left(1 \otimes \xi_{\omega}\right)\left(\iota_{\omega}\right) \in V \otimes V$ :

Proposition 4.2. In $S(V)_{A}, \kappa_{\omega}^{2}=p_{\omega}$.

Returning now to the setting of $\S 2$, recall that the symmetric bilinear form $\omega_{\varphi}=-(\varphi, \varphi) B_{\theta} \mid p_{\varphi}^{2}$ is nonsingular on $p_{\varphi}^{2}$. Call a nonassociative algebra $A$ (together with a linear injection from $k \oplus \hat{p}_{\varphi}^{2}$ into $A$ ) $\varphi$-admissible if it is $\omega_{\varphi}$-admissible. In addition to the Jordan algebra of $\omega_{\varphi}$ and the Clifford algebra of $\omega_{\varphi}$, note that by Proposition 2.1, the composition algebra $c_{\varphi}$ is $\varphi$-admissible.

Assume that $A$ is $\varphi$-admissible, let $G$ be the universal enveloping algebra of $g$, and form the nonassociative algebra $G_{A}=A \otimes G$. Identify $p_{\varphi}^{2}$ with its image in $A$.

As in [3(b), $\S \S 4$ and 5], for $\psi \in \Sigma$, let $t_{\psi} \in S^{2}\left(\left(g^{\psi}\right)^{*}\right)$ be the canonical quadratic form on $g^{\psi}$ defined by $B_{\theta}$, let $\xi_{\psi}: S\left(\left(g^{\psi}\right)^{*}\right) \rightarrow S\left(g^{\psi}\right)$ be the canonical isomorphism defined by $B_{\theta}$, let $p_{\psi}=\xi_{\psi}\left(t_{\psi}\right) \in S^{2}\left(g^{\psi}\right)$, and let $q_{\psi}=$ $2 \lambda\left(p_{\psi}\right) /(\psi, \psi) \in G$, where $\lambda$ is the symmetrization mapping from $S(g)$ to $G$. We shall apply this to the cases $\psi=\varphi$ and $\psi=2 \varphi$. If $2 \varphi \notin \Sigma$, take $t_{2 \psi}, \xi_{2 \psi}, p_{2 \psi}$, and $q_{2 \psi}$ to be zero.

Let $\iota_{2 \varphi} \in g^{2 \varphi} \otimes\left(g^{2 \varphi}\right)^{*} \simeq$ End $g^{2 \varphi}$ be the canonical element corresponding to the identity map, and let

$$
\kappa_{2 \varphi}=(1 / 2(\varphi, \varphi))\left(i_{\varphi} \otimes \xi_{2 \varphi}\right)\left(\iota_{2 \varphi}\right) \in p_{\varphi}^{2} \otimes g^{2 \varphi} \subset A \otimes G=G_{A} .
$$

Proposition 4.3. In $G_{A}, k_{2 \varphi}^{2}=-q_{2 \varphi}$.

PRoof. Since $i_{\varphi}: g^{2 \varphi} \rightarrow p_{\varphi}^{2}$ is an isometry from $2 B_{\theta}$ to $B_{\theta}$ (see $\S 2$ ), the embedding $i_{\varphi}: \mathrm{g}^{2 \varphi} \longrightarrow A$ makes $A$ admissible for the nonsingular symmetric bilinear form $\omega=-2(\varphi, \varphi) B_{\theta}$ on $g^{2 \varphi}$. Using the notation preceding Proposition 4.2, we have

$$
\begin{gathered}
\iota_{\omega}=\left(i_{\varphi} \otimes 1\right)\left(\iota_{2 \varphi}\right) \in A \otimes\left(g^{2 \varphi}\right)^{*}, \quad t_{\omega}=-2(\varphi, \varphi) t_{2 \varphi}, \\
\xi_{\omega}\left|S^{1}\left(\left(g^{2 \varphi}\right)^{*}\right)=-2(\varphi, \varphi) \xi_{2 \varphi}\right| S^{1}\left(\left(g^{2 \varphi}\right)^{*}\right)
\end{gathered}
$$




$$
\begin{gathered}
\xi_{\omega}\left|S^{2}\left(\left(g^{2 \varphi}\right)^{*}\right)=4(\varphi, \varphi)^{2} \xi_{2 \varphi}\right| S^{2}\left(\left(g^{2 \varphi}\right)^{*}\right), \\
p_{\omega}=\xi_{\omega}\left(t_{\omega}\right)=-8(\varphi, \varphi)^{3} \xi_{2 \varphi}\left(t_{2 \varphi}\right)=-8(\varphi, \varphi)^{3} p_{2 \varphi}, \\
\kappa_{\omega}=\left(1 \otimes \xi_{\omega}\right)\left(\iota_{\omega}\right)=\left(i_{\varphi} \otimes \xi_{\omega}\right)\left(\iota_{2 \varphi}\right) \\
=-2(\varphi, \varphi)\left(i_{\varphi} \otimes \xi_{2 \varphi}\right)\left(\iota_{2 \varphi}\right) \in \phi_{\varphi}^{2} \otimes g^{2 \varphi} \subset A \otimes S\left(g^{2 \varphi}\right) .
\end{gathered}
$$

Thus by Proposition 4.2,

$$
\left(\left(i_{\varphi} \otimes \xi_{2 \varphi}\right)\left(i_{2 \varphi}\right)\right)^{2}=-2(\varphi, \varphi) p_{2 \varphi} \quad \text { in } A \otimes S\left(g^{2 \varphi}\right) .
$$

But $\lambda$ is an algebra injection of $S\left(g^{2 \varphi}\right)$ into $G$. Hence applying $1 \otimes \lambda$ to the last equation gives the desired result: Q.E.D.

Recall that the center of a nonassociative algebra is the subalgebra of its nucleus consisting of the elements which commute with every element of the algebra (see [1, p. 18]). In order to show that the subalgebra of $G_{A}$ generated by $q_{\varphi}$ and $k_{2 \varphi}$ is associative, we prove the following general fact:

LEMMA 4.4. Let $B$ be a nonassociative algebra (with 1), $C$ a subalgebra of the center of $B$, and $D$ an associative subalgebra of $B$. Then the subalgebra of $B$ generated by $C$ and $D$ is associative.

PRoOF. Let $r, s, t$ be nonnegative integers, and let $x_{1} x_{2} \cdots x_{r}, y_{1} y_{2} \cdots$ $y_{s}$ and $z_{1} z_{2} \cdots z_{t}$ be nonassociative products in $B$ (with some distribution of parentheses) such that each $x_{i}, y_{i}$ and $z_{i}$ is in either $C$ or $D$. It is sufficient to prove that the associator

$$
\left(x_{1} \cdots x_{r}, y_{1} \cdots y_{s}, z_{1} \cdots z_{t}\right)=0 .
$$

We do this by induction on $r+s+t$. The result is clear if $r+s+t<3$. Assuming the result for $r+s+t<n(n \geqslant 3)$, we shall prove it for $r+s+t=n$. We may assume that $r, s, t<n$. By the induction hypothesis and standard arguments, parentheses are not necessary in any of the three products appearing in the associator. Thus since $C$ is a central subalgebra and $D$ is a subalgebra, each of these products can be reduced to the form $c d$, with $c \in C$ and $d \in D$. Hence it is sufficient to show that $\left(c_{1} d_{1}, c_{2} d_{2}, c_{3} d_{3}\right)=0$ for $c_{i} \in C$ and $d_{i} \in D$. The $c_{i}$ being central, it is sufficient to show that $\left(d_{1}, d_{2}, d_{3}\right)=0$, in view of $[1$, p. 18, formula (31)]. Since $D$ is associative, we are done. Q.E.D.

We can now prove:

Proposition 4.5. The subalgebra of $G_{A}$ generated by $q_{\varphi}$ and $\kappa_{2 \varphi}$ is associative.

Proof. Let $E$ be the (commutative) subalgebra of $G$ generated by $q_{\varphi}$ and 
$9^{2 \varphi}$, and let $B=A \otimes E$, a subalgebra of $G_{A} . E$ is clearly contained in the nucleus and the center of $B$. Let $C$ be the subalgebra of $E$ generated by $q_{\varphi}$, and $D$ the subalgebra of $B$ generated by $\kappa_{2 \varphi}$. Using Proposition 4.3 , it is easy to see that $D$ is associative, as in the proof of Lemma 4.4 , for example. Now apply Lemma 4.4. Q.E.D.

The following result is immediate:

THEOREM 4.6. Let $A$ be a $\varphi$-admissible nonassociative algebra, for example, the composition algebra $c_{\varphi}$, the Jordan algebra of $-(\varphi, \varphi) B_{\theta} \mid p_{\varphi}^{2}$, or the Clifford algebra of this bilinear form. Define $\kappa_{\varphi} \in G_{A}=A \otimes G$ as above, and let $q_{\varphi}$ and $q_{2 \varphi}$ be the quadratic elements of $G$ defined in [3(b), §5], so that $q_{\varphi}$ and $q_{2 \varphi}$ generate a polynomial algebra $P_{\varphi}$ in two variables (one variable if $2 \varphi \notin \Sigma$ ). Then $\kappa_{2 \varphi}^{2}=-q_{2 \varphi}$; the subalgebra $Q_{\varphi}$ of $G_{A}$ generated by $q_{\varphi}$ and $\kappa_{2 \varphi}$ is the (commutative, associative) polynomial algebra in these two generators (in $q_{\varphi}$ if $2 \varphi \notin \Sigma$ ), and $Q_{\varphi}=P_{\varphi} \oplus P_{\varphi} \kappa_{2 \varphi}$.

Define the conical polynomials $\zeta_{\varphi, l} \in G(l=0,1,2, \ldots)$ as follows:

$$
\zeta_{\varphi, l}=\prod_{j=1 ; j \text { odd }}^{l-1}\left(q_{\varphi}^{2}+16 j^{2} q_{2 \varphi}\right)
$$

if $l$ is even, and

$$
\zeta_{\varphi, l}=q_{\varphi} \prod_{j=2 ; j \text { even }}^{l-1}\left(q_{\varphi}^{2}+16 j^{2} q_{2 \varphi}\right)
$$

if $l$ is odd. Except for a modification when $\operatorname{dim} g^{\varphi}=1$, the $\zeta_{\varphi, l}$ (for $\varphi=-\alpha$ ) are the polynomials $\zeta_{l}$ used to describe the conical vectors in the main result (Theorem 10.1) of [3(b)] .

Our final result follows directly from Theorem 4.6:

THEOREM 4.7. In the notation of Theorem 4.6, the conical polynomial $\zeta_{\varphi, l} \in G(l=0,1,2, \ldots)$ factors in $G_{A}$ into the following $l$ "linear" factors:

$$
\zeta_{\varphi, l}=\prod_{\substack{j=-(l-1) ; \\ i \equiv l-1(\bmod 2)}}^{l-1}\left(q_{\varphi}+4 j \kappa_{2 \varphi}\right)
$$

This factorization is well defined, taking place inside the commutative associative subalgebra $Q_{\varphi}$ of $G_{A}$.

\section{REFERENCES}

1. N. Jacobson, Structure and representations of Jordan algebras, Amer. Math. Soc. Colloq. Publ., vol. 39, Amer. Math. Soc., Providence, R.I., 1968. MR 40 \#4330.

2, B. Kostant, a) On the existence and irreducibility of certain series of representations, Bull. Amer. Math. Soc. 75 (1969), 627-642. MR 39 \#7031. 
b) On the existence and irreducibility of certain series of representations, Lie Groups and their Representations, Summer School of the Bolyai János Math. Soc., edited by I. M. Gel'fand, Halsted Press, Division of John Wiley and Sons, New York, 1975, pp. 231-329.

3. J. Lepowsky, a) On the Harish-Chandra homomorphism, Trans. Amer. Math. Soc. 208 (1975), 193-218.

b) Conical vectors in induced modules, Trans. Amer. Math. Soc. 208 (1975), 219-272.

c) Existence of conical vectors in induced modules, Ann. of Math. 102 (1975), 17-40.

4. R. D. Schafer, An introduction to nonassociative algebras, Pure and Appl. Math., vol. 22, Academic Press, New York, 1966. MR 35 \#1643.

DEPARTMENT OF MATHEMATICS, YALE UNIVERSITY, NEW HAVEN, CONNECTICUT 06520 